\title{
The anal reflex elicited by cough and sniff: validation of a neglected clinical sign
}

\author{
C L H Chan, S Ponsford, M Swash
}

J Neurol Neurosurg Psychiatry 2004;75:1449-1451. doi: 10.1136/jnnp.2003.032110

See end of article for authors' affiliations

.....................

Correspondence to:

Dr M Swash, Department

of Neurology, Royal

London Hospital, London

El 1BB, UK; mswash@

btinternet.com

Received

10 November 2003

Revised 10November 2003

Accepted 14 January 2004
Background: It is unclear whether contraction of the external anal sphincter (EAS) following a voluntary cough is an integral component of the cough response itself, or a reflex response to the abdominal and pelvic floor dynamics induced by the cough. Clinical experience suggests a reflex origin for this response. Objective: To compare motor latencies for intercostal, abdominal, and EAS muscle contraction after transcranial magnetic stimulation with those following voluntary coughing and sniffing.

Methods: A needle electrode inserted into the EAS measured responses, which were confirmed by tonic electromyographic recording. Direct motor latencies from the cerebral cortex to the intercostal, rectus abdominis and EAS muscles were obtained using transcranial magnetic stimulation. Sniff and cough induced responses were also recorded in these muscles.

Results: The results suggest that EAS responses following a voluntary cough or sniff represent a polysynaptic reflex.

Conclusions: As the cough induced anal reflex response is consistent and easily elicited, its use in clinical neurological examination is appropriate. l: 1891 Rossolimo reported that brief contraction of the anal sphincter occurred in response to pricking or scratching the perianal skin or anal mucosa. ${ }^{1}$ By ablation experiments in the dog he showed that this anal response was a spinal reflex dependent on the S4 sensory and motor nerve roots. In cauda equina lesions, this response is usually absent. ${ }^{2}{ }^{3}$ By tradition, in neurological practice the anal reflex is still assessed in this way. However, contraction of the anal sphincter also occurs following a voluntary cough, a simple bedside test that is widely used in colorectal surgical practice in the assessment of faecal and stress urinary incontinence. ${ }^{4}$ It remains uncertain whether this "anal cough response" is a programmed component of the cough itself, arising from coordinated neural activity in a presumed brainstem "cough centre", thought to be responsible for contraction of the abdominal diaphragm and intercostal muscles during coughing, or is a spinal reflex resembling the classical anal reflex, occurring in response to displacement of the pelvic floor by transiently elevated intra-abdominal pressure, itself caused by the cough. To address this question we studied the abdominal intercostals and anal sphincter responses to cortical stimulation and to cough and sniff, using classical neurophysiological methods.

\section{METHODS}

A healthy male volunteer aged 43 years, without neurological or pelvic floor disorder, consented to the study following local research ethics committee approval.

\section{Responses to cough and sniff}

Active surface recording electrodes (Neuroline 700 10-K) were applied to the right seventh intercostal muscle (anterior axillary line) and right rectus abdominis muscle $2 \mathrm{~cm}$ lateral to the umbilicus. Reference surface electrodes were placed $5 \mathrm{~cm}$ lateral to each active electrode. A concentric needle electrode was inserted into the EAS at the "9 o'clock" position. Correct positioning of the EAS needle electrode was confirmed by tonic electromyographic (EMG) potentials and by maximal EMG activity during voluntary contraction of the anal sphincter. EMG activity in each muscle was recorded simultaneously using standard EMG apparatus (Keypoint; Medtronic, Denmark). Latency was taken as the first deflection from the baseline (fig 1). The subject was asked to produce a voluntary cough through a Wright peak flow meter, as an objective measure of cough force. The latency of the EMG response in the EAS following voluntary cough was measured in five recordings made at 2 minute intervals. The subject was also asked to perform a rapid voluntary inspiratory sniff and the latency of any EAS response was measured in five recordings at 2 minute intervals.

\section{Transcortical motor stimulation}

Direct motor latencies from the cerebral cortex to the intercostal, rectus abdominis and EAS muscles were obtained using transcranial magnetic stimulation (TMS) (Magstim 2000; Magstim UK) with a figure of eight coil placed at the vertex. ${ }^{5}$ The shortest latency was taken of three consistent motor responses during slight background voluntary contraction. These experiments were repeated on three separate occasions, several weeks apart. The Mann-Whitney U test was used to analyse the latency differences.

\section{Sensory studies}

The contribution, if any, of sensory input from the anal canal to the afferent pathway of the anal cough response was assessed in a separate experiment. Topical lignocaine (5\%) gel was applied to the anal canal. ${ }^{3}$ After 15 minutes, sensation in the anal canal was tested by a thermal probe at temperatures up to $50^{\circ} \mathrm{C}$. The subject was unable to detect heat even at this noxious level. The pre-anaesthetic temperature at which heat was felt in the anal canal was $39.8^{\circ} \mathrm{C}$; the perianal skin temperature was $38^{\circ} \mathrm{C}$. During anal anaesthesia the cough protocol was repeated exactly as before.

\section{RESULTS}

Consistent responses were obtained from the three muscles (fig lA). The latency of the EAS response following TMS

Abbreviations: EAS, external anal sphincter; EMG, electromyographic; TMS, transcranial magnetic stimulation 
A

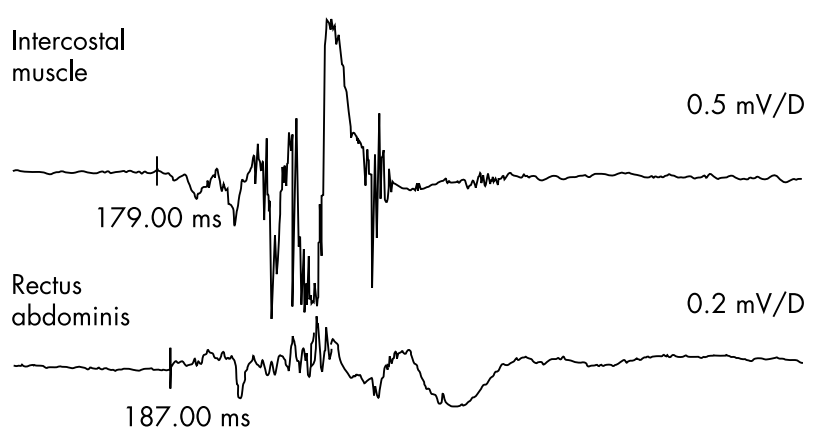

External anal
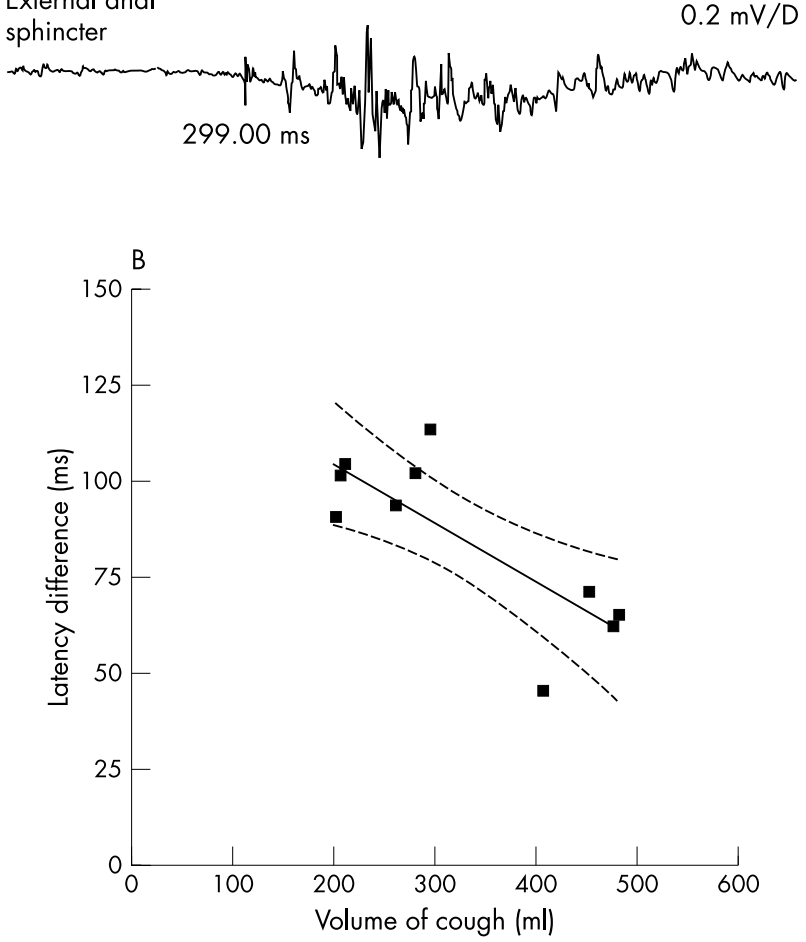

Figure 1 EMG responses in the intercostal, rectus abdominis, and external anal sphincter muscles following a voluntary cough.

ranged from 15.9 to $18.9 \mathrm{~ms}$. The mean differences between the motor latencies to the intercostal and rectus abdominis muscles following a voluntary cough (mean $8.0 \mathrm{~ms}$; confidence interval (CI) 7.0 to 9.0); and following TMS (6.7 ms; CI 5.9 to 7.6$)$ were similar $(p=0.29)$ (fig 2). However, the mean difference between the rectus abdominis and EAS motor responses was much greater following a voluntary cough (mean $93.1 \mathrm{~ms}$; CI 80.8 to 105.4: $\mathrm{p}=0.01$ ) than following TMS (mean $10.6 \mathrm{~ms}$; CI 10.0 to 11.3). A sudden voluntary sniff was followed by anal sphincter EMG activity with a latency similar to that following cough $(\mathrm{p}=0.37)$ (fig 2). The responses in the rectus abdominis and EAS muscles following cough and sniff showed similar latency differences $(p=0.52)$. There was an inverse correlation between cough force, as measured by peak air flow at the mouth, and the motor latency differences between the rectus abdominis and EAS responses $\left(r^{2}=0.63 ; p=0.006\right)($ fig $1 B)$, but the latency difference between intercostal and rectus abdominis EMG responses was unaffected by cough force (fig 2). Topical lignocaine anaesthesia of the anal canal had no effect on the motor latencies to the EAS muscle following voluntary cough $(p=0.7)$. These results were verified by

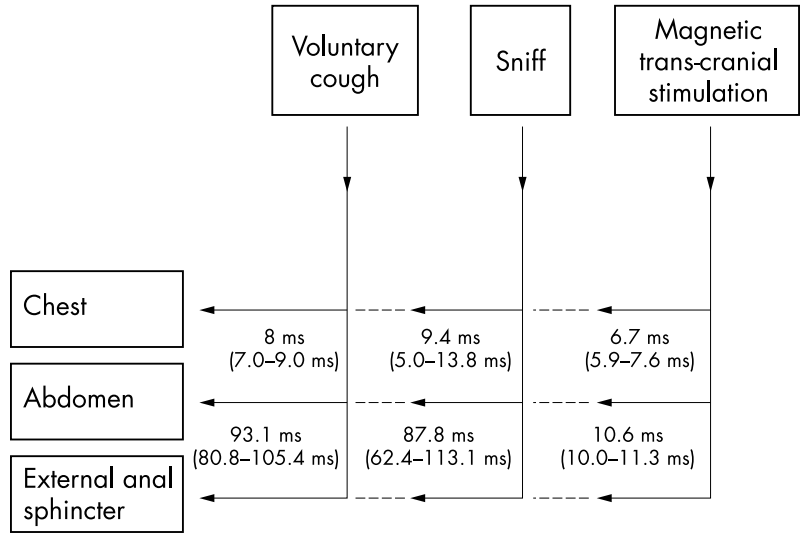

Flgure 2 Latency differences between responses at the three muscles following cough, sniff, and transcranial magnetic stimulation.

repetition of the same experimental protocol in the same normal subject 4 weeks and 6 weeks later.

\section{DISCUSSION}

Contraction of the intercostal and abdominal wall musculature occurs as part of the coordinated abrupt respiratory muscle activity that characterises both cough and sniff. We found that during a voluntary cough or sniff activation of EAS followed that of the rectus abdominis muscle, at a latency of about $90 \mathrm{~ms}$. In contrast, the latency difference between EAS and rectus abdominis responses following TMS was approximately $20 \mathrm{~ms} .{ }^{5}{ }^{6}$ With increasing cough force this latency difference decreased, but the latency difference between intercostal and rectus abdominis muscle activation remained unaltered, at $7-10 \mathrm{~ms}$, following induction by cough, sniff or TMS (fig 2). This short, consistent latency between intercostal and rectus abdominis muscle responses implies a direct spinal pathway from a central "cough centre", while the variable and much longer latency between the rectus abdominis and EAS responses is consistent with a polysynaptic reflex pathway. The fact that these relationships were unchanged after local anal anaesthesia indicates that the afferent pathway for the late EAS response we have detected does not involve sensory input from the anal mucosa. The afferent limb of the anal cough and sniff reflexes could arise from excitation of muscle spindles or other sensory receptors in pelvic floor muscles, or of Pacinian corpuscles in ligaments and fascia of the pelvic floor, ${ }^{7}$ as stimulation of these structures can induce pelvic floor contraction, although the long latency of the EAS response is somewhat against this suggestion. Sensory input from the viscera or bladder could also be involved. Slow conducting fibres (A delta) somatic afferents, backfiring of the efferent output from the rectus abdominis to the rostral "cough centre", or a non-reflexive polysynaptic spinal pathway must also be considered. However, the latter two hypotheses are unlikely in view of the modulation of the EAS latency with increasing cough force (fig 1B). A reflex origin for the EAS cough response was first suggested by Parks et al. ${ }^{8}$ Shafiq $^{9}$ reported an anal sphincter response, following sudden balloon distension of the rectum, with a latency of 38$56 \mathrm{~ms}$. The latency of the response varied inversely with the volume of the dilation. This response, unlike the cough and sniff induced responses we have studied, was abolished by high rectal neck local anaesthesia. Our electrophysiological experiments indicate that the EAS responses to sniff and cough are of reflex origin, but that the reflexogenous stimulus is not localised to the rectal mucosa. In contrast to the classical anal reflex, elicited by cutaneous stimuli, ${ }^{1-3}{ }^{7}$ we 
found no evidence of activation of the EAS following cough and sniff earlier than $70 \mathrm{~ms}^{10}$

Direct stimulation of the S3 nerve root with an implanted intraforaminal electrode is used in the neuromodulatory treatment of some urinary voiding disorders. A single stimulus applied to this nerve root induces a brief contraction of the EAS at a latency of about $90 \mathrm{~ms}$ (range 51-140 ms in nine subjects). ${ }^{11}$ This anal response has been considered, from its long latency, to be a polysynaptic reflex response, probably of local spinal origin, although a long bulbospinal pathway cannot be fully excluded by these nerve stimulation experiments. ${ }^{11}$ Observations of anal responses following midthoracic spinal cord injury show that the anal responses to cough are preserved, ${ }^{12}$ although this response is lost after dorsal rhizotomy. ${ }^{13}$ There is thus good clinical and electrophysiological evidence that the cough induced anal response is a polysynaptic spinal reflex that resembles the conventional scratch induced anal reflex.

The reflex anal responses to cough and sniff are of practical importance in clinical examination. The anal cough response is easy to evaluate by inspecting the anus during a voluntary cough, when an "anal wink" response will be seen. It is known to be unaffected by spinal cord transection in man, ${ }^{12}$ and absent in cauda equina lesions. ${ }^{3}$ The anal cough reflex adds another clinical component to the classical anal reflex. It is neither threatening nor uncomfortable for patient or examiner and we commend extension of its use from coloproctological practice to clinical neurological practice.

\section{Authors' affiliations}

C L H Chan, Academic Department of Surgery, Barts and The London School of Medicine and Dentistry, Queen Mary University of London, at the Royal London Hospital, London, UK
S Ponsford, Department of Neurophysiology, Barts and The London School of Medicine and Dentistry, Queen Mary University of London, at the Royal London Hospital, London, UK

M Swash, Department of Neurology, Barts and The London School of Medicine and Dentistry, Queen Mary University of London, at the Royal London Hospital, London, UK

Competing interests: none declared

\section{REFERENCES}

1 Rossolimo G. Der Analreflex, seine Physiologie und Pathologie. Neurologisches Centralblatt 1891;4:257-9.

2 Pedersen E. Human anal reflexes. In: Henry MM, Swash M, eds. Coloproctology and the pelvic floor. London: Butterworths, 1985:104-11.

3 Uher EM, Swash M. Sacral reflexes: physiology and clinical application. Dis Colon Rectum 1998;41:1165-77.

4 Meagher AP, Lubowski DZ, King DW. The cough response of the anal sphincter. Int J Colorectal Dis 1993;8:217-19.

5 Merton PA. Electrical stimulation through the scalp of pyramidal tract fibres supplying pelvic floor muscles. In: Henry MM, Swash M, eds. Coloproctology and the pelvic floor. London: Butterworths, 1985: 125-8.

6 Snooks SJ, Swash M. Motor conduction velocity in the human spinal cord; slowed conduction in multiple sclerosis and radiation myelopathy. J Neurol Neurosurg Psychiatry 1985;48:1135-9.

7 Swash M. Electrophysiological investigation of the posterior pelvic floor musculature. In: Pemberton JB, Swash M, Henry MM, eds. The pelvic floor London: WB Saunders, 2002:213-36.

8 Parks AG, Porter NH, Melzak J. Experimental study of the reflex mechanism controlling the muscles of the pelvic floor. Dis Colon Rectum 1962;5:407-14.

9 Pedersen E, Klemar B, Schroeder HD, et al. Anal sphincter responses after perianal electrical stimulation. I Neurol Neurosurg Psychiatry 1982;45:770-3.

10 Fowler CJ, Swinn MJ, Goodwin RJ, et al. Studies of the latency of pelvic floor contraction during peripheral nerve evaluation show that the response is reflexly mediated. J Urol 2000;163:881-4.

11 Shafiq A. Dilatation and closing anal reflexes. Acta Anat 1991;142:293-8.

12 Frenckner B. Function of the anal sphincters in spinal man. Gut 1975;16:482-9.

13 Sun WM, McDonagh R, Foster $D$, et al. Anorectal function in patients with complete spinal cord transection before and after sacral posterior rhizotomy. Gastroenterology 1995;108:990-8. 